\title{
Cutaneous Features of Anti-MDA-5 Antibody-Positive Amyopathic Dermatomyositis in a Sudanese Patient
}

\author{
Nouf Alqahtani ${ }^{a} \quad$ Majed Aleissa ${ }^{a, b, c}$ \\ aDivision of Dermatology, King Abdulaziz medical city, Ministry of National Guard Health \\ Affairs, Riyadh, Saudi Arabia; ${ }^{b}$ College of Medicine, King Saud bin Abdulaziz University \\ for Health Sciences, Riyadh, Saudi Arabia; 'King Abdullah International Medical Research \\ Center, Riyadh, Saudi Arabia
}

\section{Keywords}

Dermatomyositis · Amyopathic · Clinically amyopathic dermatomyositis · Melanoma differentiation-associated protein 5 antibody

\section{Abstract}

Clinically amyopathic dermatomyositis (CADM) is a rare form of DM characterized by unique cutaneous and pulmonary features with no muscle involvement. A subset of patients with CADM has a specific antibody known as anti-melanoma differentiation-associated protein 5 (MDA5). The systemic associations of anti-MDA-5 CADM warrant an early recognition and management to prevent fetal sequelae. It is seen more commonly in white and Asian female individuals. The clinical features of anti-MDA5 antibody-positive CADM in other ethnic groups are not well reported. Here, we describe a case of CADM with identified autoantibodies against MDA5 in a Sudanese female patient presenting with characteristic cutaneous features in association with MDA5 autoantibodies: ulcerated Gottron's papules, painful palmar papules, shawl sign, and heliotrope sign. No evidence of pulmonary or systemic involvement was identified. Treatment with prednisolone and mycophenolate mofetil was initiated. This case emphasizes the importance of keeping a high level of suspicion and to recognize the unique clinical feature of this type of DM aiding in early treatment and preventing fatal outcomes. 


\section{Introduction}

Dermatomyositis (DM) is an autoimmune disease, known to be one of the idiopathic inflammatory myopathies. Classically, it involves both skin and muscles; however, clinical subtypes without muscle involvement have been described [1]. More than 15 different autoantibodies have been linked to various clinical manifestations of DM [2]. Clinically amyopathic DM (CADM) is a rare form of DM characterized by unique cutaneous and pulmonary features with no muscle involvement. The majority of adult and juvenile CADM cases had positive autoantibodies against anti-melanoma differentiation-associated protein 5 (MDA5; previously CADM140) [1,3]. Anti-MDA5-positive CADM is seen more commonly in Asian and Caucasian female individuals in their fifth and sixth decades. The clinical features of antiMDA5 CADM in other ethnic groups is not well reported [1]. Here, we describe the cutaneous features of CADM with identified autoantibodies against MDA5 in a Sudanese patient.

\section{Case Presentation}

A 45-year-old Sudanese female patient, with no significant medical history, presented to our clinic with a 6-week history of different skin lesions: multiple painful and itchy papules on bilateral hands involving the knuckles, wrists, and palmar surfaces; multiple psoriatic plaques over the knees and elbows; and erythematous rash followed by dyspigmentation involving the chest (shawl sign) and periorbital area (heliotrope sign) (shown in Fig. 1). The lesions were worsening and increasing in number. She also complained of joint pain involving the small joints of bilateral hands, wrists, and ankles. However, she had no history of muscle pain. A comprehensive clinical history was obtained, which was not suggestive of internal malignancy or pulmonary involvement. Histopathology of the palmar lesions showed mild epidermal acanthosis, interface dermatitis with dyskeratotic keratinocytes, mild perivascular lymphocytic infiltrate, and a significant increase in mucin deposition in superficial, mid, and deep dermis (shown in Fig. 2). Blood tests and autoimmune workup revealed mildly positive ANA, high positive MDA-5 antibody, elevated ESR ( $68 \mathrm{~mm} / \mathrm{h})$, and normal creatine kinase and aldolase levels. The diagnosis of anti-MDA5 amyopathic DM was established. High-resolution computed tomography (CT) of the chest and the pulmonary function test were normal. Internal malignancy workup including a CT scan of the abdomen and pelvis was unremarkable. Treatment with prednisolone $30 \mathrm{mg}$ daily and mycophenolate mofetil (MMF) $1 \mathrm{~g}$ twice daily was initiated. Clinical improvement was demonstrated after 3 months of treatment; therefore, the dose of prednisolone was gradually tapered by $5 \mathrm{mg}$ every month, and the dose of MMF was increased to $1.5 \mathrm{~g}$ twice daily. After 7 months of therapy, skin lesions resolved with residual hyperpigmentation, with no signs of activity, and her joint pain improved significantly, and she has no constitutional symptoms, respiratory symptoms, or muscular pain. Currently, she is well controlled on $5 \mathrm{mg}$ of prednisolone once a day and $1.5 \mathrm{~g}$ of MMF twice a day.

\section{Discussion}

CADM is a rare entity that presents with cutaneous manifestations of classic DM but without muscle weakness or abnormal muscle enzymes [1]. A subset of patients with CADM has a specific antibody known as anti-MDA5. MDA5 is an RNA-specific helicase, part of innate immune system in response to viral infections, and it was first recognized in 2005 as a novel marker for CADM with distinctive clinical features [4]. Cutaneous manifestations of anti-MDA 5 CADM have been described, mainly in Asian and Caucasian populations, and include

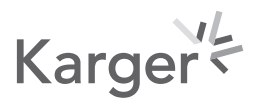




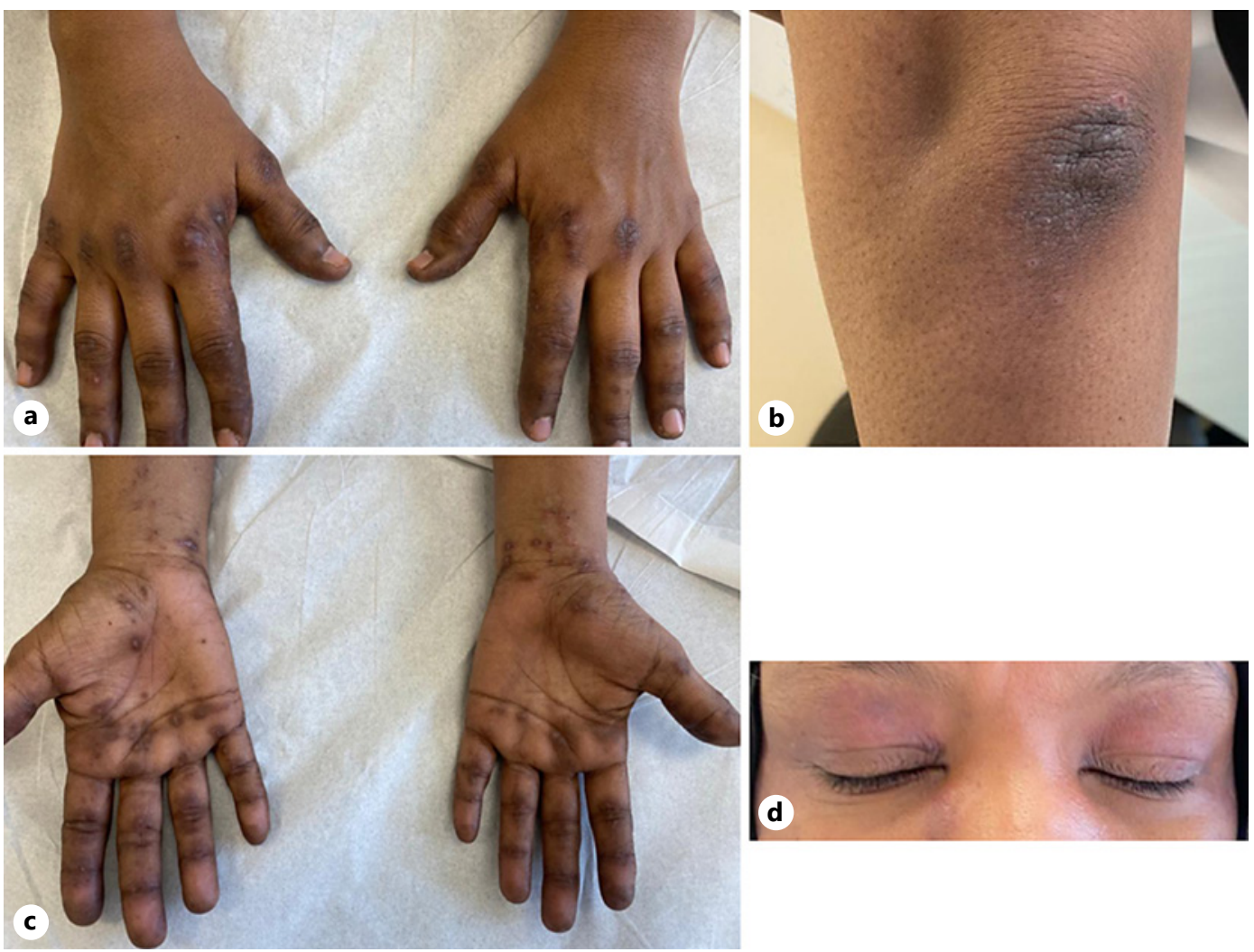

Fig. 1. a Gottron papules involving extensor surfaces of the interphalangeal joints. b Psoriatic plaques over the elbows. c Multiple painful erosive papules involving the palmar surfaces and wrists. $\mathbf{d}$ Violaceous discoloration of the upper eyelid (heliotrope sign).

ulceration at the site of Gottron's papules with or without necrosis, painful inverse Gottron's papules over the palmar surfaces, panniculitis and severe alopecia. Those patients particularly are at a higher risk of developing interstitial lung disease (ILD), which could be rapidly progressive ILD (RP-ILD), and other extracutaneous associations include arthritis, gingival pain, isolated high aldolase level, and cardiac involvement $[1,4-6]$. The systemic associations of anti-MDA 5 CADM warrant an early recognition and management to prevent fetal sequelae.

A 20-fold increased risk of developing RP-ILD was observed in those patients, in comparison to other DM patients who have negative MDA5 antibodies; the prevalence of lung involvement among these patients is estimated to be $42-100 \%$ [5]. This association is not fully understood, but endothelial injury of the pulmonary vasculature by these antibodies is a suggested theory [5]. It is associated with poor and possibly fetal outcomes, a rapidly progressive course within approximately 1-month manifested by dyspnea, low oxygen saturation, and interstitial pulmonary changes on CT [7]. The risk of malignancy with anti-MDA5 is rare; therefore, additional screening is unnecessary [1].

A suggested therapeutic regimen includes oral systemic therapy by steroids, combined with hydroxychloroquine to treat cutaneous lesions and MMF for RP-ILD. Rituximab with steroids has been shown to be effective in treating recalcitrant pulmonary disease not responding to oral agents alone. Intravenous immunoglobulin and calcineurin inhibitors were considered as third-line for recalcitrant cases [2]. The use of MMF is reported to be effective in managing ILD associated with autoimmune connective tissue diseases including CADM; it improves clinical and radiological manifestations $[8,9]$. 
Fig. 2. a Interface dermatitis with dyskeratotic keratinocytes and increase in mucin deposition in the dermis. b Significant increase in mucin deposition throughout the dermis.

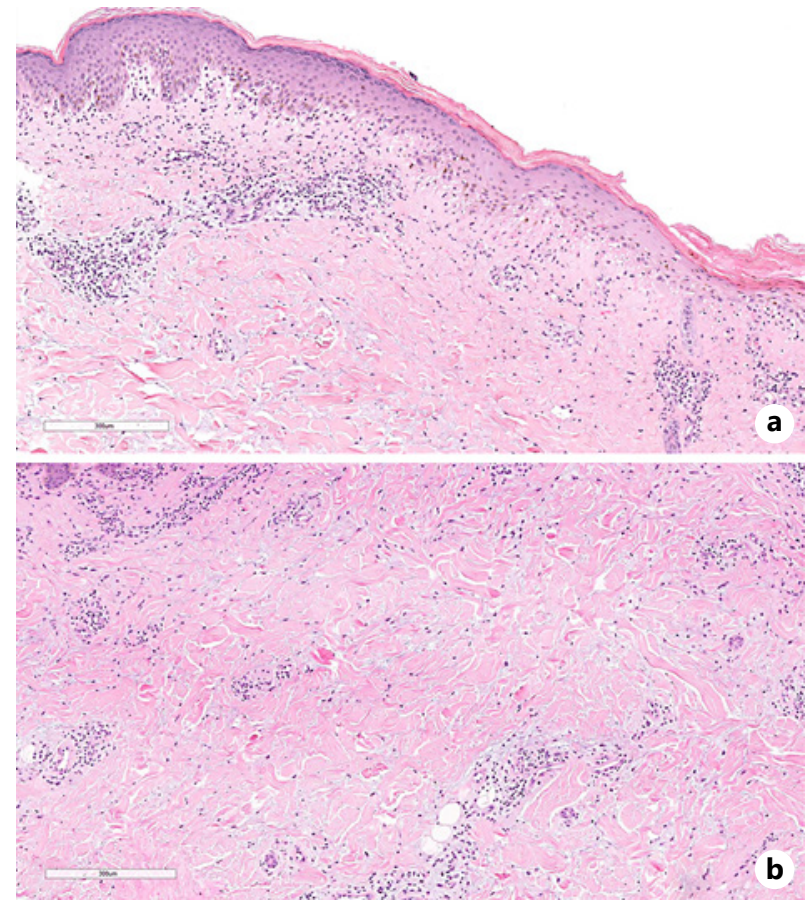

To conclude, our patient presented with the same distinctive cutaneous features seen in other ethnic groups. This case emphasizes the importance of keeping a high level of suspicion and to recognize the unique clinical feature of this type of DM aiding in early treatment and preventing fatal outcomes.

\section{Statement of Ethics}

This report complies with the Declaration of Helsinki ethical principles. Written informed consent was obtained from the patient for publication of this report and the accompanying images. Ethical approval was not required for this study in accordance with local/national guidelines.

\section{Conflict of Interest Statement}

The authors have no conflicts of interest to declare.

\section{Funding Sources}

No funding sources are relevant to the study.

\section{Author Contributions}

Nouf Alqahtani contributed to data collection and manuscript writing. Majed Aleissa involved in aonceptualization, data collection, and manuscript writing and revision. 


\section{Data Availability Statement}

All data generated or analyzed during this study are included in this article. Further inquiries can be directed to the corresponding author.

\section{References}

1 DeWane ME, Waldman R, Lu J. Dermatomyositis: clinical features and pathogenesis. J Am Acad Dermatol. 2020 Feb;82(2):267-81. Epub 2019 Jul 4. PMID: 31279808.

2 Chen L, Owens K, Murina A. Anti-MDA5 antibody-positive dermatomyositis presenting as unilateral eyelid edema. JAAD Case Rep. 2020 Jul 22;6(9):909-11. PMID: 32875043; PMCID: PMC7452317.

3 Sato S, Hoshino K, Satoh T, Fujita T, Kawakami Y, Fujita T, et al. RNA helicase encoded by melanoma differentiation-associated gene 5 is a major autoantigen in patients with clinically amyopathic dermatomyositis: association with rapidly progressive interstitial lung disease. Arthritis Rheum. 2009 Jul;60(7):2193-200. PMID: 19565506.

4 Sato S, Hirakata M, Kuwana M, Suwa A, Inada S, Mimori T, et al. Autoantibodies to a 140-kd polypeptide, CADM140, in Japanese patients with clinically amyopathic dermatomyositis. Arthritis Rheum. 2005 May;52(5): 1571-6. PMID: 15880816.

5 Kurtzman DJB, Vleugels RA. Anti-melanoma differentiation-associated gene 5 (MDA5) dermatomyositis: a concise review with an emphasis on distinctive clinical features. J Am Acad Dermatol. 2018 Apr;78(4):776-85. Epub 2017 Dec 9. PMID: 29229575.

6 Gerami P, Schope JM, McDonald L, Walling HW, Sontheimer RD. A systematic review of adult-onset clinically amyopathic dermatomyositis (dermatomyositis siné myositis): a missing link within the spectrum of the idiopathic inflammatory myopathies. J Am Acad Dermatol. 2006 Apr;54(4):597-613. Epub 2006 Jan 23. PMID: 16546580 .

7 Ideura G, Hanaoka M, Koizumi T, Fujimoto K, Shimojima Y, Ishii W, et al. Interstitial lung disease associated with amyopathic dermatomyositis: review of 18 cases. Respir Med. 2007 Jul;101(7):1406-11. Epub 2007 Mar 13. PMID: 17353121.

8 MoMorganroth PA, Kreider ME, Werth VP. Mycophenolate mofetil for interstitial lung disease in dermatomyositis. Arthritis Care Res. 2010 Oct;62(10):1496-501. PMID: 20506189; PMCID: PMC3085935.

9 Hayashi M, Kikuchi T, Takada T. Mycophenolate mofetil for the patients with interstitial lung diseases in amyopathic dermatomyositis with anti-MDA-5 antibodies. Clin Rheumatol. 2017 Jan;36(1):239-40. Epub 2016 Oct 7. PMID: 27718019. 